\title{
Multiplexing topologies and time scales: The gains and losses of synchrony
}

\author{
Sergey Makovkin, ${ }^{1}$ Anil Kumar, ${ }^{2}$ Alexey Zaikin, ${ }^{1,3}$ Sarika Jalan, ${ }^{2,4}$ and Mikhail Ivanchenko ${ }^{1}$ \\ ${ }^{1}$ Department of Applied Mathematics and Centre of Bioinformatics, \\ Lobachevsky State University of Nizhny Novgorod, Nizhny Novgorod, Russia \\ ${ }^{2}$ Complex Systems Lab, Discipline of Physics, Indian Institute of Technology Indore, Simrol, Indore, India \\ ${ }^{3}$ Institute for Women's Health and Department of Mathematics, University College London, London, United Kingdom \\ ${ }^{4}$ Centre for Bio-Science and Bio-Medical Engineering, Indian Institute of Technology Indore, Simrol, Indore, India
}

(Received 11 July 2017; published 20 November 2017)

\begin{abstract}
Inspired by the recent interest in collective dynamics of biological neural networks immersed in the glial cell medium, we investigate the frequency and phase order, i.e., Kuramoto type of synchronization in a multiplex two-layer network of phase oscillators of different time scales and topologies. One of them has a long-range connectivity, exemplified by the Erdős-Rényi random network, and supports both kinds of synchrony. The other is a locally coupled two-dimensional lattice that can reach frequency synchronization but lacks phase order. Drastically different layer frequencies disentangle intra- and interlayer synchronization. We find that an indirect but sufficiently strong coupling through the regular layer can induce both phase order in the originally nonsynchronized random layer and global order, even when an isolated regular layer does not manifest it in principle. At the same time, the route to global synchronization is complex: an initial onset of (partial) synchrony in the regular layer, when its intra- and interlayer coupling is increased, provokes the loss of synchrony even in the originally synchronized random layer. Ultimately, a developed asynchronous dynamics in both layers is abruptly taken over by the global synchrony of both kinds.
\end{abstract}

DOI: 10.1103/PhysRevE.96.052214

\section{INTRODUCTION}

Synchronization is a fundamental nonlinear phenomenon, deep in theoretical aspects and rich in real world manifestations, from living cells to power grids [1]. Phase locking (bounded phase difference) and frequency entrainment (coincidence of average frequencies) that lie in its heart can be grasped already in the dynamics of phases, neglecting amplitude effects in the weak coupling anzatz [2]. Despite a seeming simplicity and decades of investigation, its most celebrated variant, the Kuramoto model of coupled nonidentical phase oscillators [3], still makes puzzles and brings new surprises, especially for complex coupling topology [4-7].

The recent focus of attention has been taken by networks with a pronounced modular structure, also known as multilayer or multiplex, where layers (components) are networks themselves [8-14]. For example, the transport system of a country can be better represented by a multiplex network having different layers representing different modes of the transportation. Another important example where the multiplex framework is particularly useful belongs to neuroscience, where one wants to understand the origin, coordination, and function of brain rhythms of different time scales, produced in distinct interacting brain areas $[15,16]$. In this venue, considerable advance has been achieved in understanding phase synchronization in multiplex globally coupled Kuramoto ensembles with different frequency distributions [17-22]. Extensive analytical and computational studies reveal a rich picture of intra- and intercommunity synchronization regimes, including bistability, desynchronization, and chaos.

While representing the brain as a multilayer network, the topology of layers may be drastically different for example due to diverse kinds of neural connectivity in brain areas [23]. Accordingly, isolated layers can exhibit cardinally different synchronization properties. The first light on the problem was shed in Ref. [24], where a one-dimensional (1D) array was multiplexed by an offspring small-world layer, both with identical frequency distributions, however. The former layer lacks both kinds of synchronization, phase order (macroscopic Kuramoto order parameter) and frequency entrainment [25-28], while the latter shows both [29,30]. As a result, it was demonstrated that the weakly coupled 1D layer suppresses synchronization in the small-world one and enhances with strong intralayer coupling. At the same time, the 1D layer displayed clusters of frequency synchronization (groups of synchronized oscillators), instead of the network-wide synchronization. Later it was shown that the nodes even in a disconnected layer can become mutually synchronized due to coupling to a nonsynchronized layer; that, however, requires returning amplitude dynamics to the model [31].

Despite these advances, there are still many open questions. In particular, the joint effect of multiplexing diverse oscillation time scales and network topologies on synchronization is unknown [32]. This theoretical challenge, addressed in the present paper, is also inspired by the recent interest in understanding neural-glial interactions in the brain [33]. Only recently was it realized that electrically inactive glial cells (astrocytes) surrounding neurons play a key role in modulating neural synaptic communication by a bidirectional glutamate (or other gliotransmitter) mediated coupling. Diffusion of glutamate in the extracellular space dictates the local nature of neural-glial and glial-glial cells coupling, while neural synaptic connections can be long-range. On top of that, the characteristic time scales of glial chemical response is seconds, at least an order of magnitude greater than rhythmic electrical activity in neurons.

In this paper we investigate synchronization in a network of phase oscillators with multiplex topologies and time scales. To mimic to some degree topological features of real neural-glial interactions, we consider the following paradigmatic model: the faster "neural" layer is a random graph [34], locally 


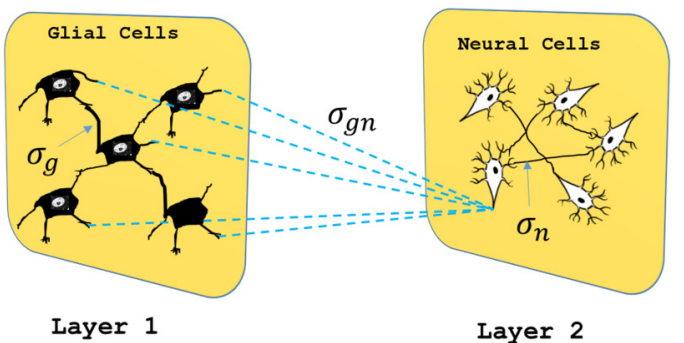

FIG. 1. Schematic diagram of multilayer network showing interlayer connections and coupling arrangements. $\sigma_{g}, \sigma_{n}$ are coupling among glial cells and neural cells, respectively. Interlink coupling strength is denoted as $\sigma_{g n}$. All links in the network are bidirectional.

coupled to the slower "glial" two-dimensional (2D) lattice; see Fig. 1. Different time scales disentangle intra- and interlayer synchronization. When "neural" coupling is weak, and the layer is not synchronized, multiplexing to the "glial" layer allows for phase order and frequency entrainment in both layers and globally, even that the isolated "glial" layer lacks coherence for all parameters. When the "neural" layer is originally synchronized, global (network-wide) phase order and entrainment are obtained as expected, provided that the "glial" lattice coupling is strong enough. Remarkably, the pathway to synchronization is mediated by the loss (or decrease) of internal synchrony in both layers, triggered by partial synchronization in the "glial" one. We find that this effect is fundamental, being manifested even in minimal models.

\section{MODEL}

We take a multilayer network in which one layer represents interactions among the glial cells and the other layer represents those of neural cells, each layer having $N \times N$ nodes. Coupling between glial cells and glial-neural interaction is mediated by glutamate diffusion and has a local character. Accordingly, we set the connections among the "glial" oscillators to form a 2D lattice. Interlayer links are such that each node in the "neural" layer is connected to its mirror and all the neighbors of the mirror node (see Fig. 1). Connections among the "neural" nodes are long-range and are modeled by an Erdős-Rényi random network [35], conditioned that each node has four edges on average, to match the connectivity of the regular layer [36].

The dynamical evolution of the nodes in this multiplex network is given by bidirectionally coupled phase oscillators

$$
\frac{d \theta_{i}}{d t}=\omega_{i}+\sum_{j=1}^{2 \times N \times N} \sigma_{i j} A_{i j} \sin \left(\theta_{j}-\theta_{i}\right),
$$

where $\theta_{i}, \omega_{i}$ are phase and natural frequency of the $i$ th oscillator, the latter taken randomly from uniform distributions $\omega_{i} \in\left[\omega_{0}^{(g, n)}-1 / 2 ; \omega_{0}^{(g, n)}+1 / 2\right]$. Characteristic time scales of neural spiking are about an order of magnitude faster than the time scale of chemical dynamics of glial cells, hence, we set the mean frequencies $\omega_{0}^{(g)}=1$ and $\omega_{0}^{(n)}=10 . A_{i j}=\{0,1\}$ are adjacency matrix elements, and coupling strength takes values $\sigma_{i, j}=\left\{\sigma_{g}, \sigma_{n}, \sigma_{n g}\right\}$, specific for interglial, interneural, and glial-neural interactions, respectively. Since glial coupling and modulation of neural activity are mediated by extracellular glutamate, we assume that related coupling coefficients are identical, $\sigma_{g}=\sigma_{n g}$, unless stated otherwise [37]. We assume open boundary conditions for both layers.

We consider two kinds and measures of synchronization. First, global phase order in the network is measured through the Kuramoto order parameter

$$
\rho=\left|\left\langle e^{i \theta}\right\rangle\right|
$$

where $0 \leqslant \rho \leqslant 1 . \rho=0$ corresponds to the case when all oscillators of the network are distributed uniformly over the circle, while $\rho=1$ represents the case when all oscillators are having same phase. Phase order in neural and glial oscillators separately is characterized by order parameters for each layer, $\rho_{n}$ and $\rho_{g}$.

Second, we calculate observed frequencies as time averages:

$$
\Omega_{j}=\frac{\theta_{j}(t)-\theta_{j}\left(t_{0}\right)}{t-t_{0}},
$$

where $t_{0}$ is taken large enough so that transient processes would essentially be over. The degree of frequency synchronization is characterized by the standard deviation of observed frequencies (3) for the whole network and each separated layer, $\Delta \Omega$, $\Delta \Omega_{g}$ and $\Delta \Omega_{n}$, respectively.

Numerical integration is implemented with a fourth-order Runge-Kutta integration scheme with the time step $\Delta t=$ 0.01 , transient time $t_{0}=1000$. Initial phases $\theta_{i}$ are taken randomly from the uniform distribution on a circle. The results are averaged over $N_{r}=10$ random realizations of frequencies and neural network wiring, depending on the system size.

\section{RESULTS}

The results show that connecting the glial and neural layers, in the way as described above, has a profound impact on synchronizability of the neural layer. First, we elaborate the case when the neural and glial layers are not coupled, which is referred to as the isolated neural and glial layer, respectively. It is known that random networks manifest both phase order and frequency entrainment, while 2D lattices lack phase order, but are capable of frequency synchronization [25-29,38]. We identify these transitions for the particular case under study. Addressing the size dependence there aids to capture the effect of network topology on synchronization. The neural layer with the random topology demonstrates the Kuramoto-type transition to phase order at $\sigma_{n} \sim 0.2$, independent on the network size [Fig. 2(a)]. In contrast, the order parameter for the glial layer of regular topology, $\rho_{g}$, progressively decays with the size of the lattice, indicating the absence of the Kuramoto transition.

Frequency characteristics demonstrate a different picture [Fig. 2(b)]. There, frequency synchronization in the glial lattice is achieved at $\sigma_{g} \sim 0.3$, without a pronounced dependence on the system size. For the neural random layer, frequency synchronization is approached somewhat earlier, however, it remains less perfect even at greater coupling.

After discussing the intrinsic properties of glial and neural layer arising due to their own interactions, we focus on the prime aim of our work, to investigate the impact of 
(a)

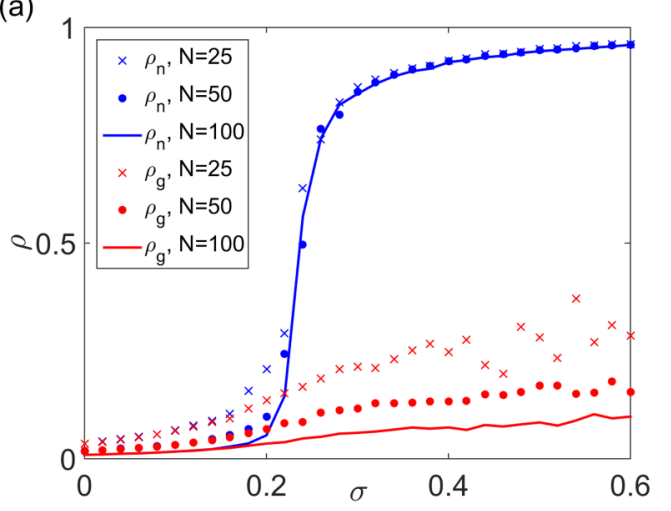

(b)

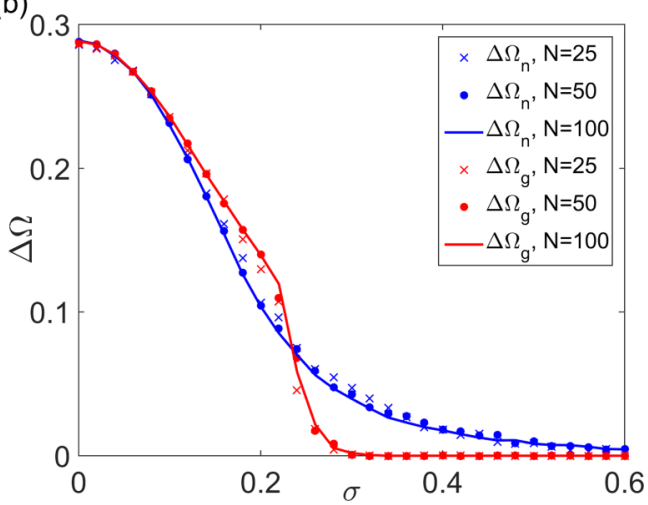

FIG. 2. Kuramoto mean-field (a) and frequency (b) synchronization in the layers of different topology in dependence on the system size, $N \times N$. Here $\sigma_{n}=\sigma_{g}=\sigma$ and the interlayer coupling is zero, $\sigma_{n g}=0$.

multiplexing on the coherent behavior of individual layers. In particular, next we investigate how glial layer interactions affect the dynamical evolution and coherent evolution neurons and vice versa.

We start with the case when the coupling in the neural layer is below the synchronization threshold, $\sigma_{n}=0.1$. Numerical results demonstrate that increasing the coupling in the glial layers and between the layers, $\sigma_{g}=\sigma_{n g}$, leads to the onset of both phase order and frequency entrainment at $\sigma_{g}=\sigma_{n g} \sim 1.1$ (Fig. 3). Remarkably, this is the result of multiplexing to the glial layer, while neither of the isolated layers exhibits phase order on its own for the chosen

(a)

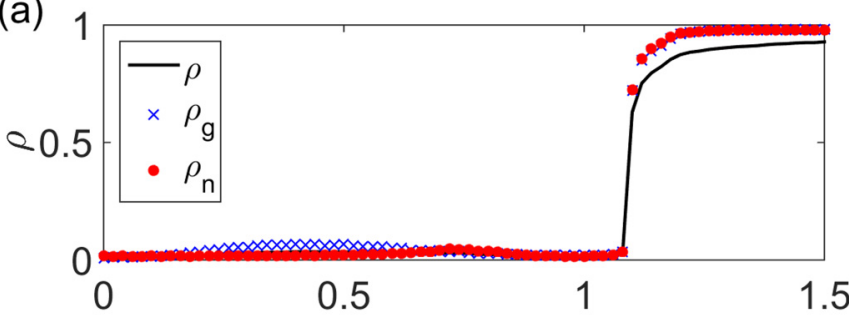

(b)

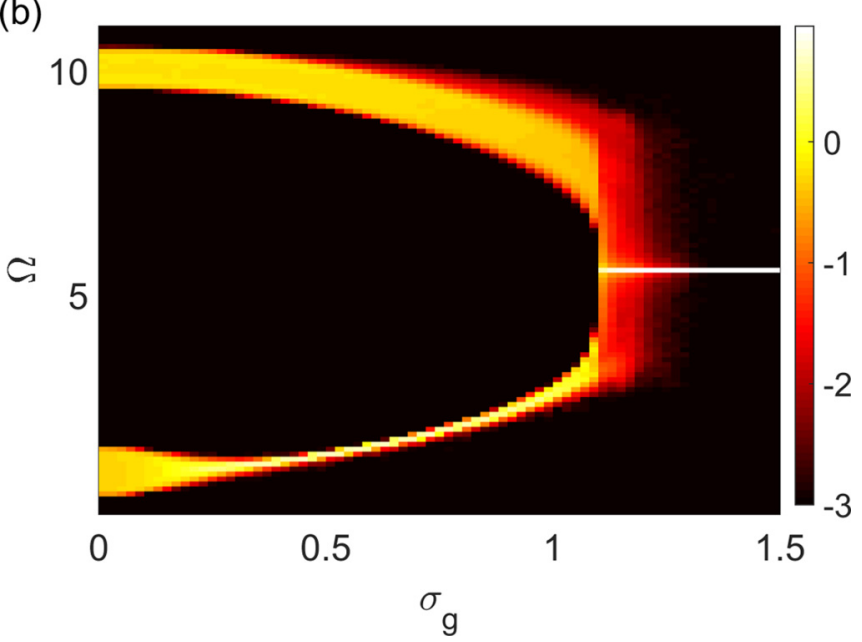

FIG. 3. Mean field (a) and color-coded $\log _{10}$ density distribution of observed frequencies (b) vs glial coupling strength, $\sigma_{g}=\sigma_{n g}$. Here $\sigma_{n}=0.1$ provides nonsynchronous oscillations in the isolated neural layer. The other parameters are $N \times N=100 \times 100$ and $N_{r}=10$. parameters, and the glial one cannot produce it for any. In this regime, individual oscillator phases in each layer are tightly grouped about respective mean phases, which are locked with a nonzero phase shift. The pathway to synchronization is surprisingly complex, because it goes through the region where both networks internally desynchronize. There is an interval $\sigma_{g}=\sigma_{n g} \sim 0.5 \ldots 1.0$, where internal frequency synchronization within the glial layer induces a noticeable widening of the frequency distribution in the neural layer.

This paradoxical loss of synchronization is even more pronounced when the neural network is above its internal synchronization threshold. This could be observed if we increase glial coupling to $\sigma_{n}=0.3$; see Fig. 4 . There one observes a gradual decrease of the neural order parameter, $\rho_{n}$, and broadening of the neural frequency distribution for $\sigma_{g}=\sigma_{n g} \gtrsim 0.3$, once a high degree of synchrony sets in the glial layer [that corresponds well to the onset of frequency synchronization in the isolated glial layer; see Fig. 2(b)]. Desynchronization develops in both layers, reaching its maximum right before a final rapid transition to global phase order and frequency synchronization at $\sigma_{g}=\sigma_{n g} \sim 1.1$.

Next we report the results of extensive calculations for subnetwork order parameters and frequency standard deviations in the coupling strengths' two-parameter plain, $\sigma_{n}, \sigma_{g}=\sigma_{n g}$ (Fig. 5) [39]. The results confirm that even for very small $\sigma_{n}$, multiplexing with the glial layer enables the onset of phase order and frequency synchronization within the neural layer and globally. Desynchronization in both layers, to a varying degree, on the route to a global synchrony is observed in the whole range of $\sigma_{n}$. Its starting point is associated with the emergence of internal frequency synchronization in the glial layer, that can break phase order and synchronization in the neural counterpart in particular.

The first interesting effect, the onset of phase order due to multiplexing with the glial layer, which does not support it itself, can be relatively easily explained. Interaction with the glial lattice, which is internally frequency synchronized (or close to this state), helps to entrain the mirror nodes in the neural layer to a certain frequency. In addition, in the strong coupling limit this leads to an additional glia-mediated neuron-neuron interaction.

The other key effect of mutual desynchronization seems to be counterintuitive. To shed some light on the mechanism 


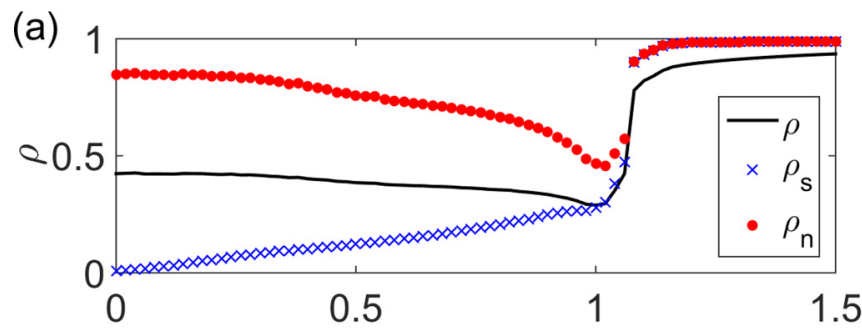

(b)

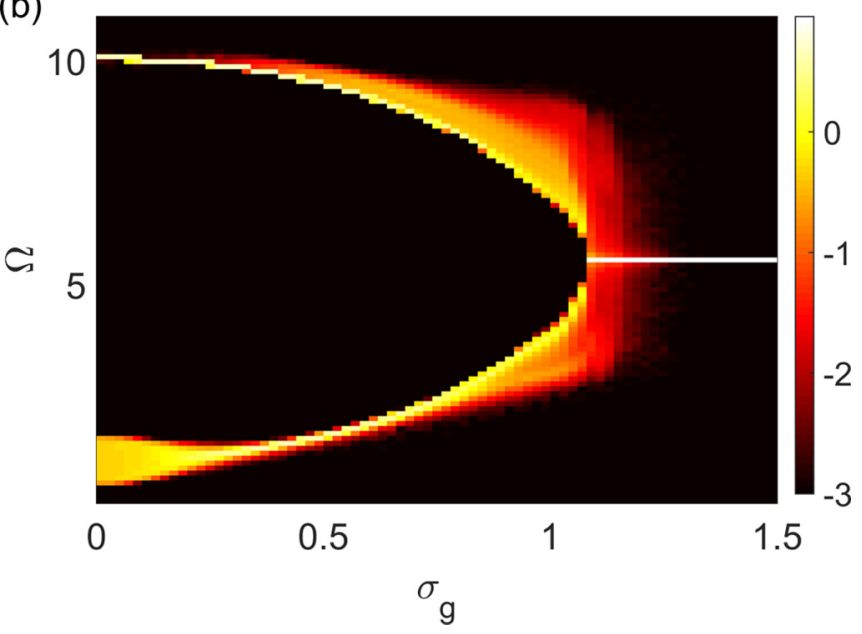

FIG. 4. Mean field (a) and color-coded $\log _{10}$ density distribution of observed frequencies (b) vs glial coupling strength, $\sigma_{g}=\sigma_{n g}$. Here $\sigma_{n}=0.3$ provides synchronous oscillations and phase order in the isolated neural layer. The other parameters are $N \times N=100 \times 100$ and $N_{r}=10$.
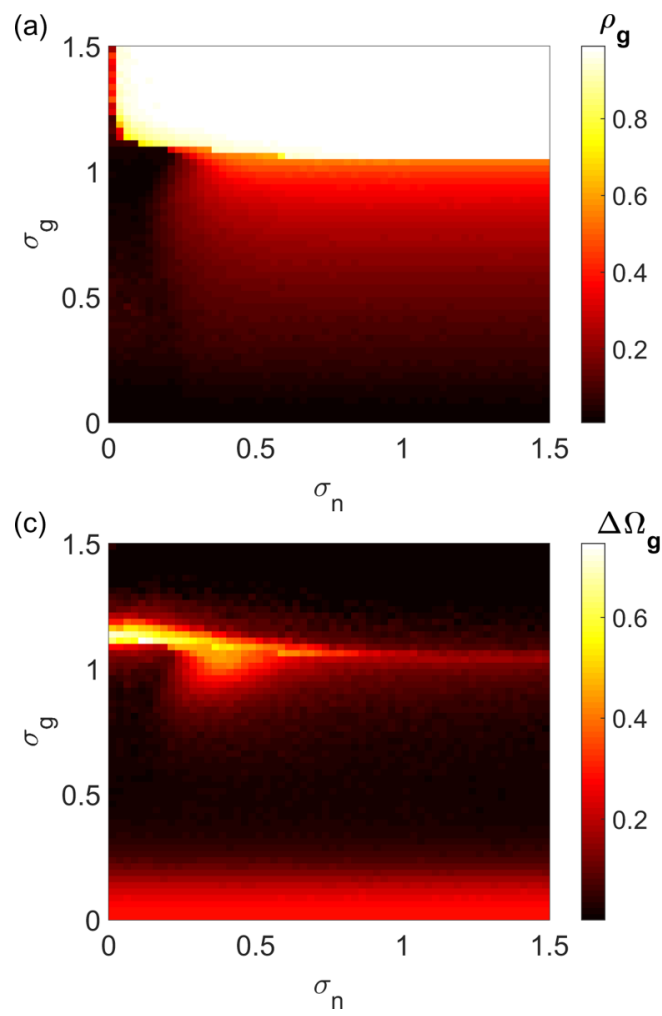

FIG. 5. Two-parameter diagrams for the color-coded subnetwork order parameters, $\rho_{g}$ and $\rho_{n}$ (a), (b) and frequency standard deviations, $\Delta \Omega_{g}$ and $\Delta \Omega_{n}(\mathrm{c}),(\mathrm{d})$. Here $N \times N=100 \times 100$.

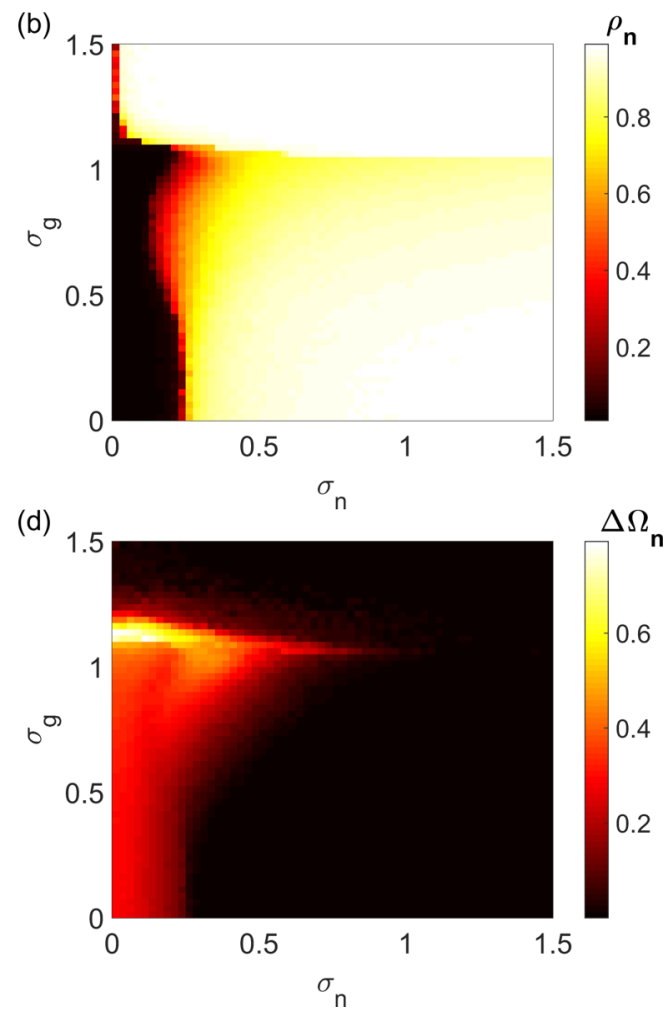

behind this behavior, we first refer to the study of entrainment of the globally coupled oscillators by an external periodic driving [40]. There it was found that for the intermediate coupling strength the internally synchronized ensemble undergoes the breakup of frequency synchronization as the driving amplitude increases. Moreover, the entrainment of the mean field oscillations appears to precede that of individual systems. While this specific feature is not readily seen in our case (see Figs. 3 and 4), the desynchronizing effect of an external periodic modulation seems quite general.

In order to pursue this observation further, we consider a minimal model of two coupled phase oscillators subject to external driving (mimicking an internally synchronized peer layer), which in the rotating frame with the driving frequency follows

$$
\begin{aligned}
& \dot{\phi}_{1}=\omega_{1}+\sin \left(\phi_{2}-\phi_{1}\right)-d \sin \phi_{1}, \\
& \dot{\phi}_{2}=\omega_{2}+\sin \left(\phi_{1}-\phi_{2}\right)-d \sin \phi_{2},
\end{aligned}
$$

where the coupling strength between the two is normalized to unity, and $d$ is the external driving strength (and frequency is 0 , formally). It is straightforward to see that in the absence of external driving and $d=0$, the oscillators are mutually synchronized, $\dot{\phi}_{1}=\dot{\phi}_{2}=\left(\omega_{1}+\omega_{2}\right) / 2$, if $\left|\omega_{1}-\omega_{2}\right| \leqslant 2$. In another limiting case, $\omega_{1}=\omega_{2}$, the oscillators are synchronized to the external driving, $\dot{\phi}_{1}=\dot{\phi}_{2}=0$, if $\left|\omega_{1,2}\right| \leqslant d$. Next, if one of the natural frequencies, $\omega_{1}$, for example, is large, $\left|\omega_{1}\right| \gg\left|\omega_{2}\right|$ and $\left|\omega_{1}\right| \gg \mid 1$, expectedly, the other can be entrained by external driving alone, if $d \gtrsim\left|\omega_{2}\right|$, such that its observed average frequency $\Omega_{2}=0$. 


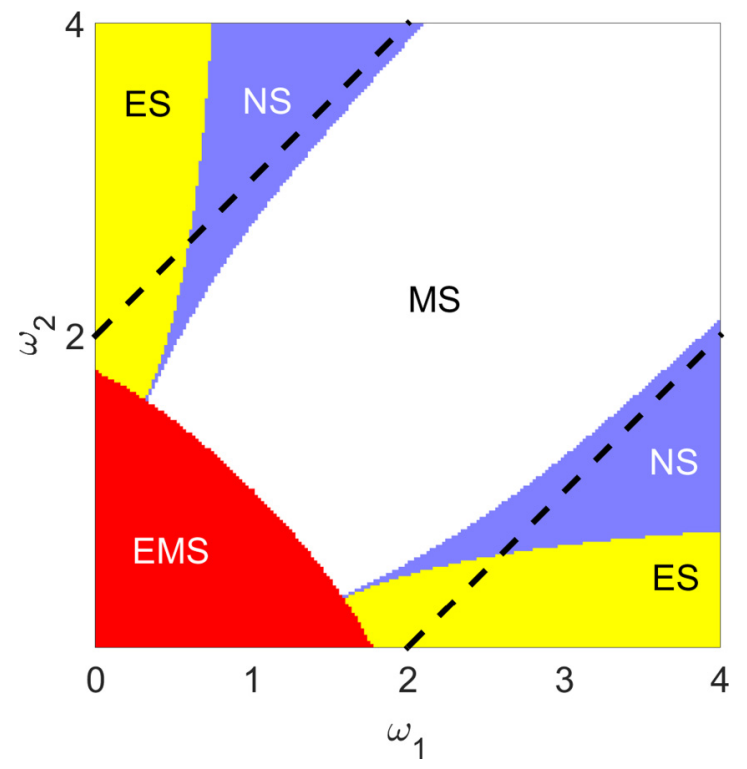

FIG. 6. Bifurcation diagram for a minimal model: mutual synchronization ("MS," white), nonsynchronized ("NS," blue), one of the oscillators externally synchronized ("ES," yellow), and both synchronized externally and mutually ("EMS," red). Here $d=1.0$. Dashed lines mark mutual synchronization region, $\left|\omega_{2}-\omega_{1}\right|<2$, in the absence of external signal, $d=0$.

Simple analytics, however, does not answer whether external driving can desynchronize the otherwise mutually synchronous oscillators, and we resort to numerics. For that the averaged observed frequencies, $\Omega_{1,2}$, are calculated (3) and compared mutually and to 0 . Figure 6 shows a typical two-parameter bifurcation diagram for the system (4), where the external driving strength is set to $d=1$ without loss of generality. Here, among the variety of synchronization regimes, we observe the regions under consideration to be within $\left|\omega_{1}-\omega_{2}\right| \leqslant 2$ (dashed lines): There any kind of synchronization is absent ("NS"), or one of the oscillators gets externally synchronized at the expense of synchronization with its peer ("ES"). We thus conclude that the intermediate desynchronization effect in the multiplex network has a basic nature.

\section{CONCLUSIONS}

Motivated by the challenges of collective dynamics of neuro-glial cell ensembles, we studied the effect of multiplexing the two networks of phase oscillators with different topology, characteristic time scales, and kinds of synchronization. One of them, the Erdós-Rényi random network, modeling the long-range neural connectivity, manifests a Kuramoto-type transition to mean-field synchronization. The other, a two-dimensional lattice, modeling local coupling in glia due to diffusion of extracellular calcium, exhibits a weaker, frequency synchronization only and lacks phase order.

In summary, we demonstrated a profound effect of multiplexing on intra- and interlayer synchronizability. When neural coupling is weak, and the layer is not synchronized, multiplexing to the glial layer allows for the phase order and frequency synchronization in both layers and globally, even when the isolated glial layer does not support the phase order at all. As expected, when the neural layer is originally synchronized, global synchronization and phase order are also obtained, provided that the glial coupling is strong enough. Remarkably, the pathway to synchronization is mediated by the loss (or decrease) of internal synchrony in both layers, following partial synchronization in the glial layer. Analysis of the simple model has shown that this effect is a manifestation of the fundamental phenomenon, present even in minimal models.

Our results open the gate to many other interesting research questions to be investigated, from broad questions of synchronization in multiplex networks with the other different topologies, extending the topology of multiplexing itself, to generalizing these effects to chaotic synchronization. Implementation of biologically realistic models of neurons and glial cells, models of synaptic coupling, glutamate diffusion, and synaptic modulation, as well as addressing the other, excitable regimes of cells, seems to be the next applicationdriven step in this research direction.

\section{ACKNOWLEDGMENTS}

This work was supported by the Russian Science Foundation Grant No. 16-12-00077 (S.M. and A.Z.), the Ministry of Education and Science of the Russian Federation Research Assignment No. 1.5586.2017/BY (M.I.), and the Department of Science and Technology project grant EMR/2016/001921 (S.J.).
[1] A. Pikovsky, M. Rosenblum, and J. Kurths, Synchronization. A Universal Concept in Nonlinear Sciences (Cambridge University Press, Cambridge, 2001).

[2] A. T. Winfree, J. Theor. Biol. 16, 15 (1967); The Geometry of Biological Time (Springer, New York, 1980).

[3] Y. Kuramoto, in International Symposium on Mathematical Problems in Theoretical Physics, Springer Lecture Notes in Physics 39 (Springer, New York, 1975), p. 420; Chemical Oscillations, Waves and Turbulence (Springer, Berlin, 1984).

[4] J. A. Acebrón, L. L. Bonilla, C. J. P. Vicente, F. Ritort, and R. Spigler, Rev. Mod. Phys. 77, 137 (2005).
[5] A. Arenas, A. Díaz-Guilera, J. Kurths, Y. Moreno, and C. Zhou, Phys. Rep. 469, 93 (2008).

[6] A. Pikovsky and M. Rosenblum, Chaos 25, 097616 (2015).

[7] F. A. Rodrigues, T. K. DM. Peron, P. Ji, and J. Kurths, Phys. Rep. 610, 1 (2016).

[8] J. Aguirre, D. Papo, and J. M. Buldú, Nat. Phys. 9, 230 (2013).

[9] S. Boccaletti, G. Bianconi, R. Criado, C. D. Genio, J. GómezGardeñes, M. Romance, I. Sendina-Nadal, Z. Wang, and M. Zanin, Phys. Rep. 544, 1 (2014).

[10] M. Kivelä, A. Arenas, M. Barthelemy, J. P. Gleeson, Y. Moreno, and M. A. Porter, J. Complex Netw. 2, 203 (2014). 
[11] R. Gutiérrez, I. Sendiña-Nadal, M. Zanin, D. Papo, and S. Boccaletti, Sci. Rep. 2, 396 (2012).

[12] P. Shinde and S. Jalan, Europhys. Lett. 112, 58001 (2015).

[13] R. Sevilla-Escoboza, I. Sendiña-Nadal, I. Leyva, R. Gutiérrez, J. M. Buldú, and S. Boccaletti, Chaos 26, 065304 (2016).

[14] C. Sarkar, A. Yadav, and S. Jalan, Europhys. Lett. 113, 18007 (2016).

[15] G. Buzsàki, Rhythms of the Brain (Oxford University Press, Oxford, 2006); N. Rosjat, S. Popovych, and S. Daun-Gruhn, Theor. Biol. Med. Model. 11, 45 (2014).

[16] M. Komarov, S. Gupta, and A. Pikovsky, Europhys. Lett. 106, 40003 (2014).

[17] M. Komarov and A. Pikovsky, Phys. Rev. E 92, 012906 (2015).

[18] S. Lück and A. Pikovsky, Phys. Lett. A 375, 2714 (2011).

[19] M. Komarov and A. Pikovsky, Phys. Rev. E 84, 016210 (2011).

[20] M. Komarov and A. Pikovsky, Phys. Rev. Lett. 110, 134101 (2013).

[21] Y. Terada and T. Aoyagi, Phys. Rev. E 94, 012213 (2016).

[22] B. Pietras, N. Deschle, and A. Daffertshofer, Phys. Rev. E 94, 052211 (2016).

[23] D. Papo, M. Zanin, J. A. Pineda-Pardo, S. Boccaletti, and J. M. Buldu, Phil. Trans. R. Soc. B 369, 20130525 (2014).

[24] J. Um, P. Minnhagen, and B. J. Kim, Chaos 21, 025106 (2011).

[25] E. Niebur, H. G. Schuster, D. M. Kammen, and C. Koch, Phys. Rev. A 44, 6895 (1991).

[26] H. Sakaguchi, S. Shinomoto, and Y. Kuramoto, Prog. Theor. Phys. 77, 1005 (1987).
[27] H. Hong, H. Park, and M. Y. Choi, Phys. Rev. E 70, 045204(R) (2004).

[28] H. Hong, H. Chatè, H. Park, and L.-H. Tang, Phys. Rev. Lett. 99, 184101 (2007).

[29] H. Hong, M.-Y. Choi, and B. J. Kim, Phys. Rev. E 65, 026139 (2002).

[30] D. J. Watts and S. H. Strogatz, Nature (London) 393, 440 (1998).

[31] L. V. Gambuzza, M. Frasca, and J. Gómez-Gardeñes, Europhys. Lett. 110, 20010 (2015).

[32] S. K. Dwivedi, M. S. Baptista, and S. Jalan, Phys. Rev. E 95, 040301(R) (2017); S. Jalan and A. Singh, Europhys. Lett. 113, 30002 (2016).

[33] M. M. Halassa and P. G. Haydon, Annu. Rev. Physiol. 72, 335 (2010).

[34] L. Muller, A. Destexhe, and M. Rudolph-Lilith, New J. Phys. 16, 105004 (2014).

[35] R. Albert and A.-L. Barabasi, Rev. Mod. Phys. 74, 47 (2002).

[36] We also checked the case of random 4-regular graphs for the neural layer to verify generality of findings.

[37] Our main observations remain valid if $\sigma_{n g} \neq \sigma_{s}$ is taken as an independent control parameter.

[38] T. Ichinomiya, Phys. Rev. E 70, 026116 (2004).

[39] We have not found traces of multistability in the studied range of parameters.

[40] O. V. Popovych and P. A. Tass, Prog. Biophys. Molec. Biol. 105, 98 (2011). 